\title{
A Case of Phlebosclerotic Ischemic Colitis: A Distinct Entity
}

Phlebosclerosis usually involves the veins of the lower extremities in the elderly, and sometimes involves the portal vein and its branches. Few reports, however, have described ischemic intestinal lesions caused by phlebosclerosis. We present here a case of ischemic intestinal lesion due to phlebosclerosis, with mesenteric venous thrombosis in a 78-year-old woman.

The patient presented with lower mild abdominal discomfort. Hematological evaluation and blood coagulation parameters showed no abnormalities. Colonoscopic inspection showed that the mucosa from the terminal ileum to the descending colon had lost its normal vascular network and appeared dark blue, with multiple small ulcers (Figure 1). The wall of the ascending colon was particularly friable, and the caliber of the lumen was extremely narrow. Radiographic examination with a barium enema revealed a rigid narrowing of the right hemicolon. Her endoscopic and fluoroscopic findings grew progressively worse, although without any serious subjective complaints. When the affected colon was resected from the terminal ileum to the descending colon, thrombosis was observed along the intestinal vasa recta. Pathological examination of the ascending colon showed a thickened purple submucosa due to marked fibrosis. The venous wall was thickened with fibrosis, hyaline sclerosis, and calcification, which contributed to the narrowing of the lumen (Figure 2). Taken together, these findings strongly suggest that the lesions were caused by an increase in mesenteric venous pressure due to phlebosclerosis.

Only a few clinically and pathologically similar cases have been reported $(1-4)$. The characteristics of the lesion in this disease could be summarized as follows: a) presence in the right hemicolon, b) dark purple mucosal appearance, c) marked thickening of the wall with an absence of the plicae semilunaris coli, and d) microscopic marked fibrous thickening of the venous wall with calcification, associated with marked submucosal fibrosis (1).

We would propose that the case reported here and those in previous reports should be considered as a distinct entity: phlebosclerotic ischemic colitis.

Y. Maruyama', F. Watanabe ${ }^{3}$, S. Kanaoka ${ }^{6}$, H. Kanamaru ${ }^{5}$,

G. Yoshino, K. Koda ', H. Hanai', E. Kaneko', I. Kinou ${ }^{2}$

' First Dept. of Medicine

${ }^{2}$ First Dept. of Pathology

Hamamatsu University School of Medicine, Hamamatsu, Japan

${ }^{3}$ Dept. of Gastroenterology

${ }^{4}$ Dept. of Pathology

${ }^{5}$ Dept. of Surgery

Fujieda City Municipal Hospital, Fujieda, Japan

${ }^{6}$ Minami Hospital, Hamamatsu, Japan

\section{References}

1. Iwashita A, Takemura S, Yamada Y, et al. Pathomorphologic study on ischemic lesions of the small and large intestine. Stomach Intest 1993; 28: 927-41.

2. Ikehata A, Hiwatashi N, Kawarada H, et al. Chronic ischemic colitis associated with marked calcifications of the mesenteric vessels. Dig Endosc 1994; 6: 355-64

3. Koyama N, Koyama H, Hanajima T, et al. Chronic ischemic colitis causing stenosis: report of a case. Stomach Intest 1991; 26: $455-60$.

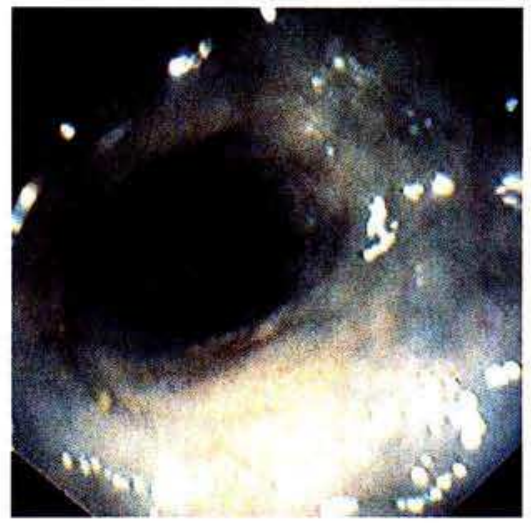

Figure 1: Colonoscopic inspection showed that the mucosa from the terminal ileum to the descending colon had lost its normal vascular network, and appeared dark blue.

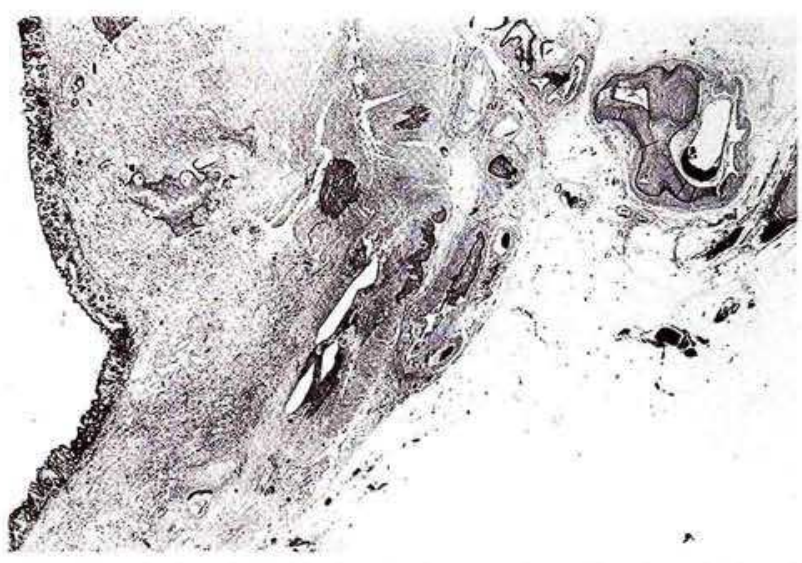

Figure 2: The histological findings in the ascending colon show thickened submucosa due to marked fibrosis. The venous wall is thickened with fibrosis, hyaline sclerosis, and calcification, causing narrowing of the lumen.

4. Hoashi T, Maeda K, Matsui T, et al. Ischemic intestinal lesion caused by phlebosclerosis with marked venous calcification: report of a case. Stomach Intest 1993; 28: 967-73.

Corresponding Author

Y. Maruyama, M.D.

First Dept. of Medicine

Hamamatsu University School of Medicine 3600 Handacho

Hamamatsu 431-31

Japan

Fax: +81-53-434-9447 